\title{
EL USO DE APRACLONIDINA EN EL DIAGNÓSTICO DEL SÍNDROME DE HORNER
}

\section{USEFULNESS OF APRACLONIDINE IN THE DIAGNOSIS OF HORNER SYNDROME}

\author{
PÉREZ-Í̃̃IGO MA ${ }^{1}$, GONZÁLEZ I I ${ }^{1}$, FERNÁNDEZ FJ ${ }^{1}$, DÍAZ $\mathrm{S}^{2}$, FERRER C ${ }^{2}$, ALIAS E $^{1}$, \\ HONRUBIA FM ${ }^{3}$
}

\begin{abstract}
RESUMEN
Casos clínicos: Se presentan cuatro casos: dos varones adultos con Síndrome de Horner clínico, diagnosticados mediante el test de apraclonidina $0,5 \%$ y el test de cocaína $4 \%$ respectivamente, y dos niños con anisocoria de los cuales, en uno de ellos el Síndrome de Horner se confirma con apraclonidina $1 \%$ y en otro el test de apraclonidina $1 \%$ descarta el Síndrome de Horner y el test de pilocarpina $0,125 \%$ confirma una pupila tónica de Adie.

Discusión: La apraclonidina al $1 \%$ y al $0,5 \%$ pueden orientar el diagnóstico de Síndrome de Horner, siendo más fáciles de obtener que la cocaína.
\end{abstract}

Palabras clave: Apraclonidina, Síndrome de Horner, anisocoria, cocaína, diagnóstico.

\begin{abstract}
Case reports: We present four cases: two males with Horner Syndrome, who were diagnosed by means of apraclonidine $0.5 \%$ and cocaine $4 \%$ tests respectively. Two children with anysocoria, one of whom had Horner Syndrome confirmed with apraclonidine $1 \%$ and the other, in whom an apraclonidine test ruled out this syndrome but in whom pilocarpine $0.125 \%$ confirmed Adie's pupil.

Discussion: Apraclonidine drops (1\% and $0.5 \%)$ may aid in the diagnosis of Horner Syndrome and are easier to obtain than cocaine (Arch Soc Esp Oftalmol 2009; 84: 105-108).
\end{abstract}

Key words: Apraclonidine, Horner Syndrome, anysocoria, cocaine, diagnosis.

\section{INTRODUCCIÓN}

Las lesiones en la vía anatómica de inervación simpática producen el llamado Síndrome de Horner. Pueden ser centrales, preganglionares o postganglionares en dependencia de su localización.

Clínicamente, se manifiestan por: ptosis leve, elevación del párpado inferior, miosis acentuada en la oscuridad, sudoración ipsilateral reducida (si es preganglionar) y heterocromía hipocrómica de iris si es congénito o de larga duración.

Las pruebas que durante años se han llevado a cabo para confirmar el diagnóstico son el test de la cocaína y el test de la hidroxianfetamina. La cocaína produce dilatación en el ojo normal pero no en el ojo afecto, por falta de noradrenalina en las terminaciones nerviosas. La cocaína inhibe la recaptación de noradrenalina en las terminaciones nervio-

\footnotetext{
Recibido: 31/5/06. Aceptado: 11/2/09.

${ }^{1}$ Doctor en Medicina. Servicio de Oftalmología. Hospital Universitario Miguel Servet. Zaragoza. España.

2 Licenciada en Medicina. Servicio de Oftalmología. Hospital Universitario Miguel Servet. Zaragoza. España.

${ }^{3}$ Doctor en Medicina. Catedrático de Oftalmología. Facultad de Medicina. Universidad de Zaragoza. Zaragoza. España.

Correspondencia:

M. ${ }^{a}$ Aránzazu Pérez Íñigo

Hospital Universitario Miguel Servet. Servicio de Oftalmología

Isabel la Católica, 1-3

50009 Zaragoza

España

E-mail: aperezinigo@ hotmail.com
} 
sas, aumentando así su efecto en condiciones normales.

La hidroxianfetamina produce dilatación en ambos ojos si la lesión es preganglionar pero no en el ojo afecto si es postganglionar, delimitando por tanto, la localización de la lesión. Ello es debido a que potencia la liberación de noradrenalina en las terminaciones postganglionares.

También se han utilizado en el pasado la adrenalina y la fenilefrina diluidas, porque la hipersensibilidad por la denervación en el Síndrome de Horner, producen una midriasis marcada, que no se observa tanto en el ojo normal $(1,2)$.

Recientemente se ha descubierto que la apraclonidina, un agonista $\alpha 2$ disponible comercialmente como hipotensor ocular, produce una dilatación en los ojos afectos de Síndrome de Horner y un efecto mínimo en ojos normales, dando lugar a una «inversión» de la anisocoria en estos pacientes, y proporcionando una confirmación diagnóstica bastante fiable (3).

Ello parece ser como consecuencia de su acción sobre los receptores $\alpha 1$, que en estos ojos afectos es mayor que en ojos normales por la denervación sufrida (2).

\section{CASOS CLÍNICOS}

\section{Adultos}

\section{Caso 1}

Varón de 31 años, intervenido de swannoma simpático apical izquierdo, tras episodios repetitivos de neumotórax.

La exploración oftalmológica posterior a la intervención fue compatible con un Síndrome de Horner izquierdo. Se realizó el test con colirio de apraclonidina $0,5 \%$ y a los 30 minutos se observó inversión de la anisocoria con leve dilatación del ojo izquierdo, disminución de la ptosis y menor elevación del párpado inferior izquierdo.

\section{Caso 2}

Varón de 46 años, fumador y ex bebedor que acudió a urgencias por leve dolor periocular derecho, ptosis y miosis de 2 meses de evolución. La exploración sistémica y neurológica fue normal y las pruebas de imagen ( Radiografía tórax, TAC cervico-torácico, RNM craneal y angio RNM cerebral) fueron normales.

No refería anhidrosis facial. La exploración oftalmológica fue compatible con Síndrome de Horner derecho, siendo el resto normal (fig. 1).

Se realizó un test con colirio de fenilefrina, siendo positiva la respuesta.

Se citó otro día para test de cocaína al $4 \%$ y se observó persistencia de miosis en ojo derecho y midriasis en ojo izquierdo, confirmándose el diagnóstico de Síndrome de Horner de etiología desconocida (fig. 2).

No se realizó el test de hidroxianfetamina para «localizar» la lesión, por no tener este colirio disponible.

\section{Niños}

\section{Caso 1}

Niña de 11 meses de edad, remitida por anisocoria con pupila izquierda más midriática.

No antecedentes de interés. Peso al nacimento: 2.800 gramos.

En la exploración destaca, ligera exotropía en ojo derecho de comienzo brusco y carácter intermitente. No ptosis. En el fondo de ojo se observan unas papilas algo pálidas. TAC craneal normal.

Se realiza el test de apraclonidina $1 \%$, y a los 30 minutos no se observan cambios en las pupilas, con lo que se descarta el Síndrome de Horner derecho.

Se cita otro día para realizar el test de pilocarpina al $0,125 \%$ y se produce miosis en ojo izquierdo con la segunda instilación, tratándose por tanto de una pupila tónica de Adie.

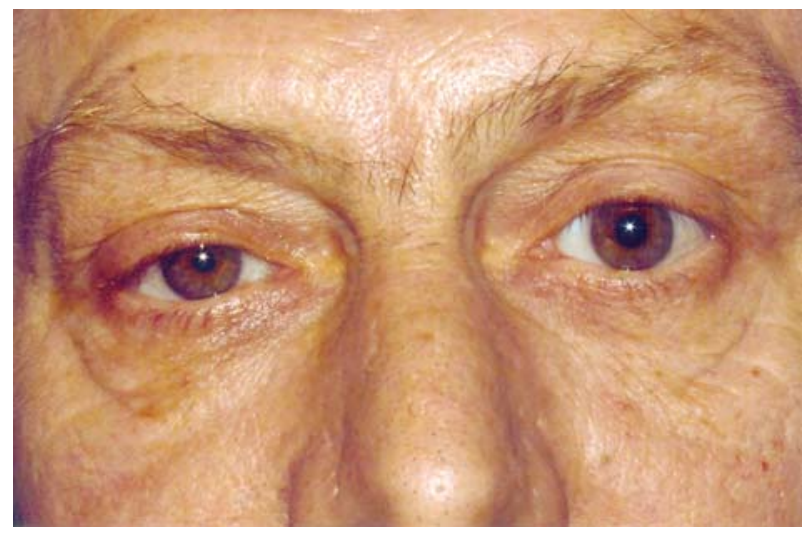

Fig. 1: Síndrome de Horner derecho. 


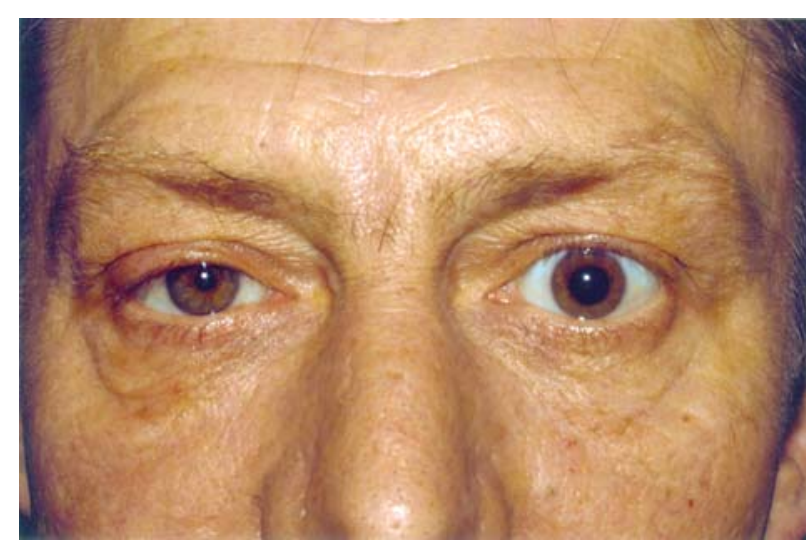

Fig. 2: Tras colirio de cocaína 4\%, se dilata el ojo no afecto, y permanece miótico el ojo derecho.

\section{Caso 2}

Niño de 4 meses remitido por ptosis y miosis en ojo izquierdo. Antecedentes: Parto por cesárea (nalgas). Nacido con comisura labial desviada (posible alteración del nervio facial en el nacimiento).

Exploración oftalmológica compatible con Síndrome de Horner izquierdo: ptosis, miosis, hipocromía de iris y anisocoria que aumenta en la oscuridad (fig. 3).

Se realiza el test de apraclonidina $1 \%$, y a los 30 minutos se invierte la anisocoria (fig. 4). Se confirma el diagnóstico de Síndrome de Horner por posible alteración del plexo braquial en el parto.

Controles neurológicos normales hasta la fecha.

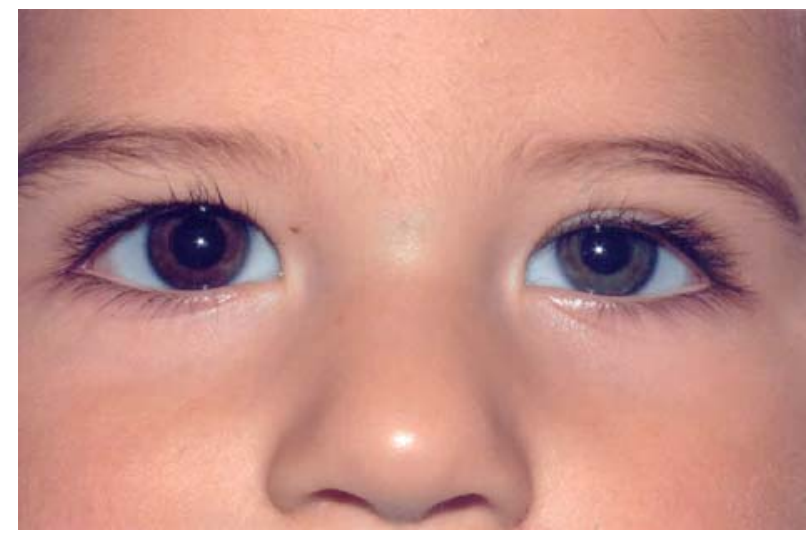

Fig. 3: Síndrome de Horner izquierdo de larga evolución: Ptosis, miosis e hipocromía de iris.

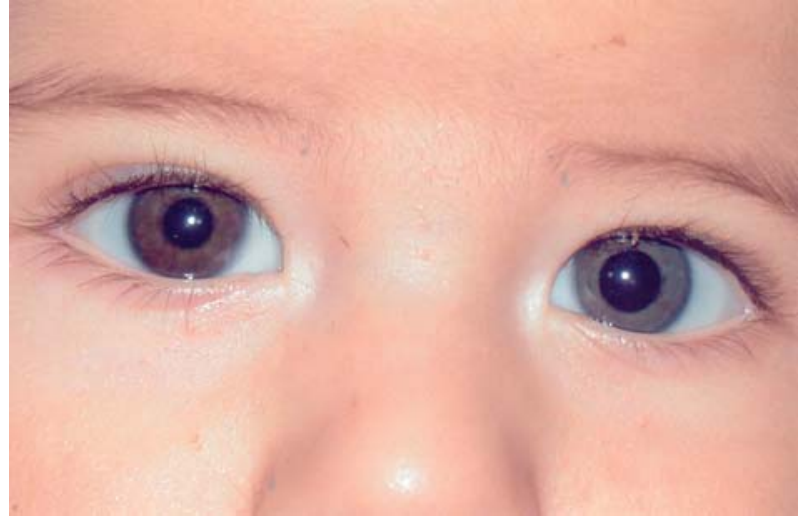

Fig. 4: Tras colirio de apraclonidina al 1\% se invierte la anisocoria y disminuye la ptosis.

\section{DISCUSIÓN}

La apraclonidina al $1 \%$ y al $0,5 \%$, pueden ser utilizadas en primera instancia para orientar el diagnóstico de posibles casos de Síndrome de Horner (3), tanto en adultos como en niños (4), sin problemas para su obtención ni efectos secundarios destacables hasta el momento. Este hecho supone una ventaja importante sobre el test de la cocaína, debido a la más fácil disponibilidad de la apraclonidina, $y$ a que se trata de un test de efecto positivo sobre el ojo afecto.

En estos casos hemos utilizado ambas concentraciones $(0,5 \%$ y $1 \%)$ indistintamente por ser casos vistos en distintos departamentos y por ser la forma que estaba disponible en ese momento. Si realmente ambas concentraciones ponen de manifiesto la inversión de la anisocoria con la misma eficacia, habría que optar por la apraclonidina al 0,5\% pues tendría potencialmente menos efectos secundarios, sobretodo en el caso de los niños (4).

Los efectos secundarios con una instilación son poco probables, en cualquier caso se podrían producir con más frecuencia: hiperemia y escozor a nivel local, sequedad de boca, y más raramente somnolencia, cefalea, mareos, alteraciones del ritmo cardiaco y alteraciones digestivas. Nosotros no hemos objetivado reacciones adversas en ninguno de los casos.

Si la lesión de la vía simpática es muy reciente, podríamos encontrarnos con un falso negativo por falta de hipersensibilidad, y en este caso habría que recurrir a los test clásicos (5). 


\section{BIBLIOGRAFÍA}

1. Kardon R. Are we ready to replace cocaine with apraclonidine in the pharmacologic diagnosis of Horner syndrome? J Neurophthalmol 2005; 25: 69-79.

2. Morales J, Brown SM, Abdul-Rahim AS, Crosson CE. Ocular effects of apraclonidine in Horner syndrome. Arch Ophthalmol 2000; 118: 951-954.
3. Brown SM, Aouchiche R, Freedman KA. The utility of $0.5 \%$ apraclonidine in the diagnosis of Horner syndrome. Arch Ophthalmol 2003; 121: 1201-1203.

4. Bacal DA, Levy SR. The use of apraclonidine in the diagnosis of Horner syndrome in pediatric patients. Arch Ophthalmol 2004; 122:276-279.

5. Freedman KA, Brown SM. Topical apraclonidine in the diagnosis of suspected Horner syndrome. J Neurophthalmol 2005; 25: 83-85. 